\title{
COMPUTING THE WALLS ASSOCIATED TO BRIDGELAND STABILITY CONDITIONS ON PROJECTIVE SURFACES*
}

\author{
ANTONY MACIOCIA ${ }^{\dagger}$
}

\begin{abstract}
We derive constraints on the existence of walls for Bridgeland stability conditions for general projective surfaces. We show that in suitable planes of stability conditions the walls are bounded and derive conditions for when the number of walls is globally finite. In examples, we show how to use the explicit conditions to locate walls and sometimes to show that there are no walls at all.
\end{abstract}

Key words. Bridgeland stability, moduli spaces, projective surfaces, walls.

AMS subject classifications. Primary: 14F05; Secondary: 14J60, 18E30, 18E40.

Introduction. Throughout this paper we let $X$ be a smooth complex projective surface. The notion of stability condition for a triangulated category (and, in particular, for the derived category of coherent sheaves on $X$ ) was introduced by Bridgeland (see [Bri07]). Bridgeland went on in [Bri08] to construct explicit families of stability conditions on K3 and abelian surfaces. Arcara and Bertram ([ABL07]) then showed that these examples work for all smooth complex projective surfaces. We shall be exclusively interested in these examples and their associated walls. We will call these basic (Bridgeland) stability conditions.

There are various reasons for studying such stability conditions now well documented in the literature. For example, the preservation of stability under FourierMukai transforms correspond to the non-existence of walls. The Göttsche conjecture and Donaldson-Thomas invariants are (conjecturally) related to wall crossing formula in such stability spaces.

One particular use is to help us to compute explicit moduli spaces of objects in the derived category of a variety. At a boundary of the space of stability conditions the stability condition becomes the more familiar one of (twisted) Gieseker stability. We can construct these spaces using techniques of Geometric Invariant Theory and often they are related to other moduli spaces such as moduli spaces of connections satisfying certain differential equations. But for fixed characteristic classes, the space of stability conditions is divided into chambers and once we cross a wall into a different chamber the objects representing points of the corresponding moduli space need not be torsion-free sheaves or indeed be sheaves at all. In order to better understand these moduli spaces (including their existence) our first step is to locate the walls separating the chambers. These walls arise from a combination of numerical constraints and constraints coming from the hom sets of objects in the derived category. Indeed, understanding better which walls can exist should help us understand the derived category and its sets of maps. The numerical constraints turn out to be essentially quadratic in suitable coordinates in the stability manifold $\operatorname{Stab}(X)$ and so are (real) quadrics. If we restrict to certain real half-planes in $\operatorname{Stab}(X)$ given by pairs $\left(\mathcal{A}_{\beta, \omega}, Z_{\beta, \omega}\right)$ (see section 1.2 for a detailed description of this) then these are conic sections with centres on the boundary of the half-planes. We will prove that, provided we choose the plane correctly, these are heavily constrained to be nested. By this we

\footnotetext{
*Received November 28, 2012; accepted for publication December 14, 2012.

$\dagger$ Department of Mathematics and Statistics, The University of Edinburgh, The King's Buildings, Mayfield Road, Edinburgh, EH9 3JZ, U.K. (A.Maciocia@.ed.ac.uk).
} 
mean that the conics lie entirely inside each other so that they can never intersect or lie in two halves of the plane divided by a straight line (see Figure 1 in the text for a typical example). We prove:

Theorem 3.1 (Bertram's Nested Wall Theorem). Let $X$ be a smooth complex projective surface. Pick an ample $\omega \in \mathrm{NS}(X)$ and a real class $\beta \in \operatorname{NS}_{\mathbb{R}}(X)$. Then for any class $v \in \mathrm{N}(X)$ representing the Chern character of a $\mu_{\omega}$-semistable sheaf or torsion sheaf on $X$ there is a half-plane $\Pi=\Pi_{\beta, \omega, 1}$ in $\operatorname{Stab}(X)$ which contains the basic Bridgeland stability condition $\left(\mathcal{A}_{\beta, \omega}, Z_{\beta, \omega}\right)$ such that the intersection of the walls with $\Pi$ are nested.

In particular, if the Picard Group $\rho(X)=1$ the walls in the space of basic stability conditions are nested.

This theorem was conjectured by Aaron Bertram and there was already a lot of evidence for it in the Picard rank 1 case on $\mathbb{P}^{2}$ and on abelian surfaces (see [MM11] for the abelian surface case). The key ingredients are the Bogomolov inequality and the Hodge Index Theorem (just as in [ABL07]) applied to $v$.

The proof uses only the numerical constraints (we will call the resulting walls pseudo-walls in what follows) and actually proves the stronger result that the radii of the circles depends only on the position of the centre of the circles. It also follows from the analysis that given a Chern character $v$ there exists a real number $C_{0}$ such that every wall must cross the line $s=C_{0}$. This is particularly helpful to help us locate walls by providing bounds on the Chern classes of destabilizing objects. The number $C_{0}$ is given by the formula

$$
\frac{c_{1}(v) \cdot \omega}{r(v) \omega^{2}}-\sqrt{F},
$$

where $F$ is given by Equation 2.1. $F$ depends only on $v$ and the choice of plane $\Pi_{\beta, \omega}$. In the special case of Picard rank $1, F=\Delta(v) /\left(r(v)^{2} \omega^{2}\right)$, where $\Delta(v)$ is the discriminant of $v$. More generally, without the condition on the Picard rank, we show that when $F=0$ there can be no non-trivial walls at all (see Theorem 3.6). In other words, when $F=0$, a Bogomolov critical $\mu_{\omega}$-semistable sheaf must always be $\mu_{Z}$-stable for any basic Bridgeland stability condition $(\mathcal{A}, Z)$ in the plane $\Pi_{\beta, \omega}$.

Using our constraints on the walls and the fact that walls must correspond to actual destabilizing objects we show that the radii of the walls are always bounded above (and we give explicit computable bounds). More precisely, we shall show:

TheOREM 3.11. Let $\beta$ be any class in $N(X)_{\mathbb{R}}, \omega$ an ample class and $u \in \mathbb{R}$. Fix a Chern character $v$ of a $\mu_{\omega}$-semistable sheaf or a torsion sheaf. In the half-plane $\Pi_{\beta, \omega, u}$ there are real numbers $C_{0}$ and $R_{0}$ such that all of the walls corresponding to $v$ are contained in the semi-circle with centre $\left(C_{0}, 0\right)$ and radius $R_{0}$. In particular, the radii of the walls are bounded above by $R_{0}$.

Together with local finiteness of the walls we can then give a new more direct proof of a result in [LQ11] which shows that for a given $\beta$ and $\omega$, there is some $M \in \mathbb{R}$ such that there are no mini-walls (these are just the intersection of walls with rays $t>0$ in our planes) for $t \in[M, \infty)$. We also show that for all but one ray in our plane the number of walls is actually globally finite.

We finish with a number of examples. Firstly, we show how to use $C_{0}$ to quickly show that, for certain Chern classes $v$, walls cannot exist. We then look again at the [MM11] situation of an irreducible principally polarized abelian surface and rank 1 
sheaves. We also consider the same situation but for a product abelian surface and this allows us to construct an example to show that for other choices of plane, the walls do not nest. We also show how to conclude global finiteness results for walls for special choices of variety. We finish with a result which gives an example of how to quickly deduce vanishing results for walls. This final example looks at the 6 dimensional O'Grady space of rank 2 charge 2 stable bundles on an abelian surface which famously provides an exceptional compact simply-connected holomorphic symplectic manifold. We show that there are no walls for the associated Chern character.

\section{Stability conditions and their walls.}

1.1. The numerics of a surface. Let $X$ be a smooth complex projective surface and let $D(X)=D(\operatorname{Coh} X)$ denote its bounded derived category of coherent sheaves. We let $\mathrm{N}(X)$ denote the numerical Grothendieck group of $X$. For a coherent sheaf $A$ on $X$ we let $v(A)$ denote its image in $A$. This can be extended to the derived category by defining $v(a)=\sum_{i}(-1)^{i} v\left(A^{i}\right)$, where $a \in D(X)$ and we use the convenient abbreviated notation $A^{i}=H^{i}(a)$.

To be more definite we shall identify $\mathrm{N}(X)$ with $\mathbb{Z} \oplus \mathrm{NS}(X) \oplus \mathbb{Z}\left[\frac{1}{2}\right]$, where the factors correspond to $r(A), c_{1}(A)$ and $\operatorname{ch}_{2}(A)[X]$. We shall freely write $v(a)=$ $\left(r(a), c_{1}(a), \operatorname{ch}_{2}(a)[X]\right)$ corresponding to this decomposition and abuse notation by writing $\operatorname{ch}_{2}(E)=\operatorname{ch}_{2}(E)[X]$. Note that the rank of $\mathrm{NS}(X)$ is the Picard number $\rho(X)$ of $X$. We denote the intersection pairing on $\mathrm{NS}(X)$ by $(c, d) \mapsto c \cdot d$. Its signature is $(1, \rho(X)-1)$. This is extended to a pairing of signature $(2, \rho(X))$ on $\mathrm{N}(X)$ by $(v, w) \mapsto v_{1} w_{3}+v_{3} w_{1}+v_{2} \cdot w_{2}$.

For any $\mathbb{Z}$-module $M$ we write $\mathrm{N}_{M}(X)=\mathrm{N}(X) \otimes M$ and $\mathrm{NS}_{M}(X)=\mathrm{NS}(X) \otimes M$. In particular, when $M=\mathbb{Q}, \mathbb{R}$ or $\mathbb{C}$. We abuse notation and write $\alpha \in \mathrm{NS}_{M}(X)$ for the image of some $\alpha \in N S_{N}(X)$, where $M$ is an $N$-module. Suppose $\omega \in \operatorname{NS}(X)$ is an ample class and given any non-zero element $\beta$ of $\mathrm{NS}_{\mathbb{R}}(X)$ we write $\beta=b \omega+\gamma$ for the unique element $\gamma=\gamma(\beta)$ of $\mathrm{NS}_{\mathbb{R}}(X)$ which is orthogonal to $\omega$. We let $\tilde{\gamma}$ denote a choice of element of $\mathrm{NS}(X)$ which maps to $\gamma$ in $\mathrm{NS}_{\mathbb{R}}(X)$. We can play the same game in $\mathrm{NS}_{\mathbb{Q}}(X)$ but in that case there is a canonical choice for $\tilde{\gamma}$ given by $n \gamma$ for the least positive integer $n$. This trick is also used in Kawatani [Kaw11] to analyse slightly different stability conditions.

For a fixed ample class $\omega \in \operatorname{NS}(X)$ we let $\mu_{\omega}(v)=c_{1}(v) \cdot \omega / r(v)$ for any vector $v \in \mathrm{N}_{R}(X)$, interpreting it as $\pm \infty$ when $r(v)=0$. We let $\mu_{\omega}(a)=\mu_{\omega}(v(a))$ for an object $a \in D(X)$. As is well known, any sheaf $E \in \operatorname{Coh}(X)$ has a unique HarderNarasimhan filtration by $\mu_{\omega}$-semistable sheaves $\left\{E_{i}\right\}_{i=1}^{n}$ with $\mu_{\omega}\left(E_{i}\right)>\mu_{\omega}\left(E_{i+1}\right)$. We write $\mu_{\omega}^{+}(E)=\mu_{\omega}\left(E_{1}\right)$ and $\mu_{\omega}^{-}(E)=\mu_{\omega}\left(E_{n}\right)$.

1.2. Distinguished subvarieties of $\operatorname{Stab}(X)$. Now, fix an ample class $\omega \in$ $\mathrm{NS}(X)$ and another class $\beta \in \mathrm{NS}_{\mathbb{R}}(X)$. Then we can define an abelian subcategory $\mathcal{A}_{\beta, \omega}$ of $D(X)$ by the following tilt with respect to $\operatorname{Coh}(X)$ :

$$
\begin{aligned}
& T_{\beta, \omega}=\left\{E \in \operatorname{Coh}(X): E \text { is torsion or } \mu_{\omega}^{-}(E)>\beta \cdot \omega\right\} \\
& F_{\beta, \omega}=\left\{E \in \operatorname{Coh}(X): E \text { is torsion-free and } \mu_{\omega}^{+}(E) \leqslant \beta \cdot \omega\right\} .
\end{aligned}
$$

Notice that, for any $t>0, \mathcal{A}_{\beta, t \omega}=\mathcal{A}_{\beta, \omega}$. We can make these abelian subcategories into a Bridgeland stability condition by defining

$$
Z_{\beta, \omega}(v)=-\exp (-\beta-i \omega) \cdot v
$$

in $\mathrm{N}_{\mathbb{C}}(X)$. The fact that $\operatorname{Im} Z \geqslant 0$ and equal to zero only if $\operatorname{Re} Z<0$ is an easy exercise using the Hodge Index Theorem and the Bogomolov inequality. The details 
are discussed in $[\mathrm{ABL} 07, \S 2]$ and also [BM11, §4]. In fact, we do not seem to need the Harder-Narasimhan property in our analysis. We shall refer to these as basic stability conditions. To be explicit note that

$$
Z_{\beta, \omega}((r, \theta, z))=-z+\theta \cdot \beta-\frac{r}{2}\left(\beta^{2}-\omega^{2}\right)+i(\theta-r \beta) \cdot \omega .
$$

We can use this to describe subvarieties of $\operatorname{Stab}(X)$. Firstly $\left(\mathcal{A}_{\beta, \omega}, Z_{\beta, \omega}\right)$ is a point of $\operatorname{Stab}(X)$ (actually, a non-trivial fact). We can also consider the set $\left(\mathcal{A}_{\beta, \omega}, Z_{\beta, t \omega}\right)$ as a ray, denoted $\Xi_{\beta, \omega}$, in $\operatorname{Stab}(X)$. Note that this starts at the open boundary of $\operatorname{Stab}(X)$ (corresponding to $t=0$ ). If $\beta \neq 0$, we can also consider the "plane" (or more properly, "half-plane") $\left\{\left(\mathcal{A}_{s \beta, \omega}, Z_{s \beta, t \omega}\right): s \in \mathbb{R}, t>0\right\}$. This turns out to be hard to describe in general and we modify this to a family of planes as follows. Write $\beta=b \omega+\gamma$ as above and then consider the (usually) half 3-space of stability conditions

$$
\Omega_{\beta, \omega}=\left\{\left(\mathcal{A}_{s \omega+u \gamma, \omega}, Z_{s \omega+u \gamma, t \omega}\right): s, u \in \mathbb{R}, t>0\right\} .
$$

We can now consider the $u$-indexed family of planes given as the subspaces of $\Omega_{\beta, \omega}$ where $u$ is fixed. We shall denote each of these planes by $\Pi_{\beta, \omega, u}$.

NOTES 1.1.

1. If $\gamma=0$ (so $\beta \propto \omega$ ) then $\Omega_{\beta, \omega}$ is just a half-plane $\Pi_{\beta, \omega, 0}=\Pi_{\omega, \omega, 0}$. If $\rho(X)=1$ then $\gamma$ must be zero.

2. The ray $\Xi_{0, \omega}$ is contained in $\Pi_{\omega, \omega, 0}$.

3. More generally, if $b=\beta \cdot \omega=0$ but $\beta \neq 0$ then $\Omega_{\beta, \omega}=\Omega_{\beta+\omega, \omega}$ is still three dimensional.

4. Suppose $\alpha \in \mathrm{NS}_{\mathbb{Q}}(X)$ is non-zero and orthogonal to both $\gamma$ and $\omega$. Then $Z_{\beta, \omega}(v)=Z_{\beta, \omega}(v+\alpha)$.

EXAmple 1.2. Consider the case of a product abelian surface $(\mathbb{T}, \omega)=\left(\mathbb{T}_{1} \times\right.$ $\left.\mathbb{T}_{2}, \ell_{1}+\ell_{2}\right)$. Assume NS(T) $=\left\langle\ell_{1}, \ell_{2}\right\rangle$. Then, over $\mathbb{Q}$ we have $\mathrm{NS}_{\mathbb{Q}}(\mathbb{T})=\left\langle\omega, \ell_{1}-\ell_{2}\right\rangle$. Then $\Omega_{\ell_{1}-\ell_{2}, \omega}$ contains all of the possible basic stability conditions.

1.3. Walls and pseudo-walls. Given a stability condition $(\mathcal{A}, Z)$, we say that an object $a \in \mathcal{A}$ is semistable if for all proper injections $b \rightarrow a$ in $\mathcal{A}$ we have $\phi(b) \leqslant \phi(a)$, where $\phi(b)=\arg (Z(v(b)))$. Equivalently, we can use the slope function:

$$
\begin{aligned}
\mu_{Z}(b) & =-\frac{\operatorname{Re} Z(v(b))}{\operatorname{Im} Z(v(b))} \\
& =\frac{z-\theta \cdot \beta+\frac{r}{2}\left(\beta^{2}-\omega^{2}\right)}{(\theta-r \beta) \cdot \omega},
\end{aligned}
$$

where $v(b)=(r, \theta, z)$ and $Z=Z_{\beta, \omega}$.

Now fix a non-zero class $v \in \mathrm{N}(X)$ and for the following definition we assume that $v=v(a)$ for some object $a$ of $D(X)$.

Definition 1.3. We say that a class $w \in \mathrm{N}(X) \backslash\langle v\rangle$ is critical for $v$ if each of the following condition hold:

There is a $\sigma \in \operatorname{Stab}(X)$ and objects $a, b \in \mathcal{A}_{\sigma}$ with an injection $b \rightarrow a$

in $\mathcal{A}_{\sigma}$ such that $v(a)=v$ and $v(b)=w$ and $\mu_{Z}(a)=\mu_{Z}(b)$. 
If $w$ is critical for $v$ then we write $W_{w}^{v} \subset \operatorname{Stab}(X)$ for the set of such $\sigma$ and call it the wall corresponding to $w$. We drop the $v$ from the notation if it is understood.

Notes 1.4. If there is no $a \in D(X)$ such that $v(a)=v$ then there can be no critical $w$. Generally, we assume that $v$ is the Chern character of some object.

If a wall exists then it must be a real codimension 1 subspace of $\operatorname{Stab}(X)$. The complement of the union of all of the walls is a disjoint union of chambers with the property that if for any $\sigma_{0}$ in a chamber and object $a$ is $\sigma_{0}$-stable, then it remains $\sigma$-stable for all $\sigma$ in the same chamber. One would hope that there is a good coarse moduli scheme for such objects and crossing a wall would correspond to a surgery on these moduli spaces. We do not pursue the details here but instead use this as motivation to provide information about finding such walls. To help us do this we introduce a weaker notion of pseudo-wall.

Definition 1.5. We say that a class $w \in \mathrm{N}(X) \backslash\langle v\rangle$ (respectively, $w \in \mathrm{N}_{\mathbb{R}}(X) \backslash$ $\langle v\rangle)$ is $\mathbb{Z}$-critical (respectively, $\mathbb{R}$-critical) for $v$ if there is a stability condition $\sigma$ such that $\mu_{Z}(w)=\mu_{Z}(v)$.

REMARK 1.6. It is clear that a wall is also an integral pseudo-wall and an integral pseudo-wall is also a real pseudo-wall. But the converses are generally false.

REMARK 1.7. The set of pseudo-walls (real or integral) do not depend very much on $X$.

REMARK 1.8. Note that we will be interested in these purely numerical constraints on the existence of walls (embodied in the pseudo-walls) which are well defined for any $v \in \mathrm{N}_{M}(X)$ but in the applications we will always want $v$ to be a valid class corresponding to either a $\mu_{\omega}$-semistable sheaf $E$ or a torsion sheaf $E$. The Bogomolov inequality then implies that $c_{1}(v)^{2} \geqslant 2 r(v) \operatorname{ch}_{2}(v)$. Note also that if $v=v(E)$ then $E \in T_{s \omega+u \gamma, \omega}$ and so either $r(E)=0$ or $\mu_{\omega}(E)>s \omega^{2}$.

Definition 1.9. We say that $v \in \mathrm{N}_{M}(X)$ (for any $M$ ) is a Bogomolov class if it satisfies the Bogomolov inequality:

$$
c_{1}(v)^{2} \geqslant 2 r(v) \operatorname{ch}_{2}(v) .
$$

REMARK 1.10. Finally, in this section observe that twisting $v$ by $\omega$ shifts the walls and pseudo-walls by a unit to the right.

2. Purely numerical constraints. Now fix a Bogomolov class $v=(x, \theta, z)$ and an ample class $\omega$ in $\operatorname{NS}(X)$. Assume for the moment that $\rho(X)>1$ and fix a class $\gamma \in \mathrm{NS}_{\mathbb{Q}}(X)$ which is orthogonal to $\omega$. Write $\mathrm{NS}_{\mathbb{Q}}(X)=\langle\omega\rangle \oplus\langle\gamma\rangle \oplus\langle\omega, \gamma\rangle^{\perp}$. Decompose $\theta=y_{1} \omega+y_{2} \gamma+\alpha$, where $\alpha \in\langle\omega, \gamma\rangle^{\perp}$. If $\rho(X)=1$ then we can usually just let $\gamma=0$. Let $Z=Z_{s \omega+u \gamma, t \omega}$. We shall also abbreviate $\omega^{2}=g$ and $\gamma^{2}=-d$. Note that $d \geqslant 0$ by the Hodge Index Theorem and $d=0$ if and only if $\gamma=0$. Then

$$
\mu_{Z}(v)=\frac{z-s y_{1} g+u y_{2} d+\frac{x}{2}\left(s^{2} g-u^{2} d-t^{2} g\right)}{\left(y_{1}-x s\right) g t} .
$$


Let $w=\left(r, c_{1} \omega+c_{2} \gamma+\alpha^{\prime}, \chi\right)$. Then

$$
\mu_{Z}(w)-\mu_{Z}(v)=\frac{\left(y_{1}-x s\right)\left(\chi-s c_{1} g+u c_{2} d+\frac{r}{2}\left(s^{2} g-u^{2} d-t^{2} g\right)\right)}{-\left(c_{1}-r s\right)\left(z-s y_{1} g+u y_{2} d+\frac{x}{2}\left(s^{2} g-u^{2} d-t^{2} g\right)\right)} .
$$

The numerator $\times 2$ then equals (collecting terms in $s$ and $t$ ):

$$
\begin{aligned}
& g\left(x c_{1}-y_{1} r\right) s^{2}-2\left(\chi x-r z+c_{2} u d x-y_{2} u d r\right) s+ \\
& \quad g\left(x c_{1}-y_{1} r\right) t^{2}-2 z c_{1}+2 c_{2} u d y_{1}+x u^{2} d c_{1}-2 y_{2} u d c_{1}+2 \chi y_{1}-r u^{2} d y_{1} .
\end{aligned}
$$

Now assume $\mu_{\omega}(v) \neq \mu_{\omega}(w)$ (so we can divide by $\left.c_{1} x-r y_{1}\right)$. Completing the squares we can write this as

$$
g\left(x c_{1}-r y_{1}\right)\left((s-C)^{2}+t^{2}-D-C^{2}\right),
$$

where

$$
\begin{gathered}
C=\frac{x \chi-r z+u d\left(x c_{2}-r y_{2}\right)}{g\left(x c_{1}-r y_{1}\right)} \\
D=\frac{2 z c_{1}-2 c_{2} u d y_{1}-x u^{2} d c_{1}+2 y_{2} u d c_{1}-2 \chi y_{1}+r u^{2} d y_{1}}{g\left(x c_{1}-r y_{1}\right)} .
\end{gathered}
$$

This shows that in the plane $\Pi_{\gamma, \omega, u}$ using coordinates $(s, t)$, the walls are semicircles with centres $(C, 0)$ and radii $R=\sqrt{D+C^{2}}$.

Notation 2.1. We will generally use $C$ and $R$ to denote the centre and radius of any pseudo-wall in question. To make the parameters clearer we will occasionally write $C=C_{v}(w)$ and $R=R_{v}(w)$ emphasizing the fact that these are functions of a variable $w$ indexed by a parameter $v$.

REMARK 2.2. In the case where $\mu_{\omega}(w)=\mu_{\omega}(v)$ there are no square terms and the wall is a line of the form $s=y_{1} / x$ which, for completeness, we can view as a circle of infinite radius.

We now want to show that the dependence of the radii on $w$ is entirely through the centres.

Lemma 2.3. With the notation as above, if $x \neq 0$,

$$
D=\frac{u d\left(2 y_{2}-u x\right)+2 z}{g x}-\frac{2 y_{1}}{x} C
$$

Proof. Simplify $D+\frac{2 y_{1}}{x} C$. The details are left to the reader.

When $x=0$ we do not have to consider the radii:

LEMMA 2.4. If $x=0$ and $y_{1}>0$ then $C=\frac{z+d u y_{2}}{g y_{1}}$. In particular, the centre is independent of $w$.

We leave the elementary proof as an exercise for the reader. 
REMARK 2.5. So, if $x>0$, the radius is

$$
R=\sqrt{D+C^{2}}=\sqrt{\left(C-\frac{y_{1}}{x}\right)^{2}-F}
$$

where

$$
F=\frac{d}{g}\left(u-\frac{y_{2}}{x}\right)^{2}+\frac{1}{x^{2} g}\left(y_{1}^{2} g-y_{2}^{2} d-2 x z\right) .
$$

When $v=v(E)$, for some sheaf $E$, the last term in $F$ is $\frac{1}{x^{2} g}$ times $\Delta(E)-\alpha^{2}$, where $\Delta(E)$ is the discriminant of $E$. If we assume $v$ is a Bogomolov class then this is nonnegative $\left(\alpha^{2} \leqslant 0\right.$ by the Hodge Index Theorem). Since, $d \geqslant 0$ by the Hodge Index Theorem we have $F \geqslant 0$ for all $u$.

2.1. Real constraints. We can now prove:

Proposition 2.6. Suppose $v$ is a Bogomolov class. Then in $\Pi_{\gamma, \omega, u}$, the real pseudo-walls are nested. By nested we mean that if $W_{1}$ and $W_{2}$ are two distinct (semicircular) pseudo-walls in $\Pi_{\gamma, \omega, u}$ then either $W_{1}$ is entirely contained in the interior of the semi-circle $W_{2}$ or vice-versa. In particular, distinct pseudo-walls cannot intersect and one pseudo-wall cannot be entirely outside any other pseudo-wall.

Proof. When $x=0$ this follows immediately from Lemma 2.4 as the centres of the circles are fixed. Note that $y_{1}>0$ unless $v$ is the class of a sheaf supported in dimension 0 . So we suppose that $x \neq 0$. The result will follow from Lemma 2.3 if we can show that $C+R$ and $R-C$ are both monotonic increasing (or decreasing) as functions of $C$. Note that

$$
\frac{d}{d C}(C \pm R)=1 \pm \frac{1}{R}\left(C-\frac{y_{1}}{x}\right) .
$$

But $F \geqslant 0$ for all $u$ by the Remark 2.5 . Hence, $R \leqslant\left|C-y_{1} / x\right|$ and so if $C \neq y_{1} / x$, $\left|\frac{d}{d C}(C+R)\right| \geqslant 0$ with equality if and only if $F=0$. Similarly $\left|\frac{d}{d C}(C-R)\right| \geqslant 0$ for all $C \neq y_{1} / x$ but has the opposite sign to the $C+R$ case. If $F=0$ then

$$
\{C+R, C-R\}=\left\{2 C-\frac{y_{1}}{x}, \frac{y_{1}}{x}\right\}
$$

and again we are done.

Note that two pseudo-walls may be coincident. In fact, there will typically be continuous families of $w$ giving coincident pseudo-walls (by solving $C=C_{0}$ for $w$ ).

REMARK 2.7. Now $R>0$ exactly when

$$
\left(C-\frac{y_{1}}{x}\right)^{2}>F \geqslant 0 .
$$

So either $C>y_{1} / x+\sqrt{F}$ or $C<y_{1} / x-\sqrt{F}$. But, by Remark 1.8, we also have $y_{1} / x>s$ and so $C<y_{1} / x$. Then $\frac{d}{d C}(C-R) \geqslant 2$. Note also that $R \rightarrow \infty$ as $C \rightarrow-\infty$. Consequently, we have that any point $(s, t) \in\left\{(a, b): a<y_{1} / x, b>0\right\}$ is contained in at most one real pseudo-wall (see Figure 1). 


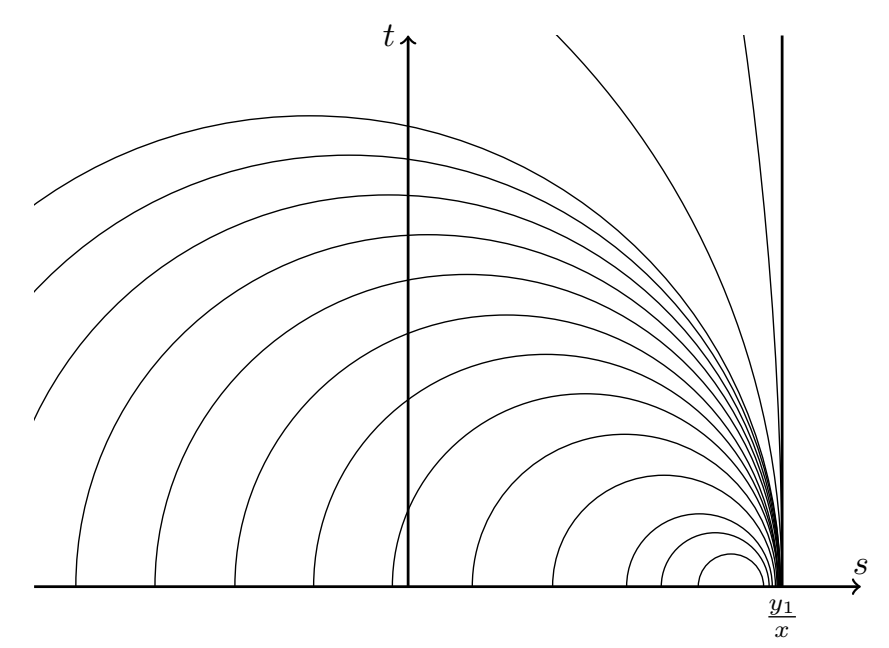

FIG. 1. Typical real pseudo-walls

Corollary 2.8. For $F>0$, any pseudo-wall must intersect $s=C_{0}$, where

$$
C_{0}= \begin{cases}\frac{y_{1}}{x}-\sqrt{F} & \text { if } x>0 . \\ \frac{z+d u y_{2}}{g y_{1}} & \text { if } x=0 \text { and } y_{1}>0\end{cases}
$$

Remark 2.9. This is particularly useful when trying to locate walls. For example, we must have

$$
0<c_{1}-r s<y_{1}-x s
$$

for the characteristic classes of a destabilizing object of an object $e \in \mathcal{A}_{s}$. Since any such object must destabilize for some $t$ when $s=C_{0}$ we obtain the following useful bounds on $c_{1}$ :

$$
r C_{0}<c_{1}<y_{1}+(r-x) C_{0} .
$$

See Example 4.1 and Proposition 4.6 below.

REMARK 2.10. Using the result of Lo and Qin [LQ11] which says that for each ray there is an upper bound on the radius we can now deduce that bound is uniform in $s$. In the next section we will give an explicit such bound but it will also follow that the bound is not uniform in $u$ (and we give an example in a later section to show that it can be unbounded).

\section{Applications.}

3.1. Bertram's Nested Wall Theorem. We can use these technical results to quickly prove:

TheOREM 3.1. Let $X$ be a smooth complex projective surface. Pick an ample $\omega \in \mathrm{NS}(X)$ and a real class $\beta \in \mathrm{NS}_{\mathbb{R}}(X)$. Then for any class $v \in \mathrm{N}(X)$ representing 
the Chern character of a $\mu_{\omega}$-semistable sheaf or a torsion sheaf on $X$ there is a halfplane $\Pi=\Pi_{\beta, \omega, 1}$ in $\operatorname{Stab}(X)$ which contains the basic Bridgeland stability condition $\left(\mathcal{A}_{\beta, \omega}, Z_{\beta, \omega}\right)$ such that the intersection of the walls with $\Pi$ are nested. nested.

In particular, if $\rho(X)=1$ the walls in the space of basic stability conditions are

Proof. We decompose $\beta=b \omega+\gamma$ as usual with $\gamma \cdot \omega=0$. Then Proposition 2.6 implies that the real pseudo-walls are nested. Since the actual walls are a subset of these walls, these must be nested as well.

One immediate corollary is that each chamber is path connected to the $t=0$ axis:

Corollary 3.2. For any $\beta$ and $\omega$, the moduli space of S-equivalence classes of $\mu_{Z}$-semistable objects with Chern character $v$ for $Z=Z_{s \omega+u \beta, t \omega}$ is isomorphic to a moduli space of $\mu_{Z}$-semistable objects with the same Chern character $v$ for some $t$ arbitrarily small.

This shows that Bertram's "sliding down the wall" trick used in [MM11] always works. In the case of abelian surfaces and K3 surfaces this implies that such moduli spaces are projective varieties and represent the appropriate moduli functor (see [MM11] and [MYY11b] for special cases of this).

3.2. A lower bound on the centres. We have seen in Remark 2.7 that there is an upper bound for the centres of the walls. We now show that the centres of actual walls are also bounded below as well (at least for a large class of $v$ ). This follows theoretically from the work of Lo and Qin ([LQ11, Theorem 1.1(ii)]) who show that in each ray there are no walls for $t$ sufficiently large. Their construction is not constructive and our aim here is to use the Nested Wall Theorem to compute explicit bounds.

We prove this in a series of lemmas. For these technical lemmas we need some parameters from the surface. Let $\tilde{g}=\operatorname{gcd}_{E}\left(c_{1}(E) \cdot \omega\right)$ over all sheaves $E$. Then $g / \tilde{g}=g^{\prime}$ is an integer and $\tilde{y}_{1}=y_{1} g^{\prime}$ is always an integer (even though $y_{1}$ may not be). Initially we shall assume that $x=r(v) \neq 0$ and deal with the $x=0$ case afterwards. For any such $v$ we let $p=\operatorname{gcd}\left(r(v), c_{1}(v) \cdot \omega / \tilde{g}\right)=\operatorname{gcd}\left(x, \tilde{y}_{1}\right)$ in the notation of the previous section. What we do in the lemma is to show that if we choose $s$ sufficiently close to $y_{1} / x$ we can ensure that there are no subsheaves of $E$ in $A_{s}$. We have to work harder to show that there can be no $\mu_{Z}$-destabilizing objects of rank bigger than $r(E)$. When $p=1$ it turns out that the same bound works but we have to go closer to $y_{1} / x$ if $p>1$.

The bound is constructed as follows. Using Euclid's algorithm, consider the set of pairs of integers $(m, n)$ such that $n \tilde{y}_{1}-m x=p$. We assume $n>0$. Fix some $\left(m_{0}, n_{0}\right)$ and then observe that any other pair is given by $(m, n)=\left(m_{0}+y^{\prime} \lambda, n_{0}+x^{\prime} \lambda\right)$ for some integer $\lambda$, where $y^{\prime}=\tilde{y}_{1} / p$ and $x^{\prime}=x / p$. Note that the ratio $m /\left(g^{\prime} n\right)$ tends to $y_{1} / x$ from below as $n \rightarrow \infty$ (whatever sign $y_{1}$ takes). So given any positive real number $\xi$, we can pick some $\lambda$ such that the set

$$
\left\{(j, k) \in \mathbb{Z}^{2}: \frac{m}{n}=\frac{m_{0}+\lambda y^{\prime}}{n_{0}+\lambda x^{\prime}}<\frac{j}{k}<\frac{\tilde{y}_{1}}{x}, \operatorname{gcd}(j, k)=1,0<k \leqslant \xi x\right\}
$$

is empty. Let us, for the moment, denote the least choice of such $m$ and $n$ for a given $\xi$ by $m_{0}$ and $n_{0}$ and define the set

$$
P_{\xi}=\left\{(m, n): n \tilde{y}_{1}-m x=p \text { and }|m| \geqslant\left|m_{0}\right|, n \geqslant n_{0}, n>\frac{p}{g^{\prime}} \sqrt{\frac{g}{\Delta}}\right\},
$$


where $\Delta=g y_{1}^{2}-d y_{2}^{2}-\alpha^{2}$. This last mysterious condition on $n$ often imposes no constraint but it will ensure that any wall which is sufficiently near $y_{1} / x$ must also cross $m /\left(g^{\prime} n\right)$ whenever $(m, n) \in P_{\xi}$.

Now suppose $E$ is a torsion-free sheaf with $v(E)=v$ and $s<y_{1} / x$. Then $\mu_{\omega}(E)=\frac{m \tilde{g}}{n}+\frac{\tilde{g}}{n x^{\prime}}=\frac{\tilde{y}_{1} \tilde{g}}{x}=\frac{y_{1} g}{x}>s g$ and so it satisfies a necessary condition for $E \in \mathcal{A}_{s \omega+u \gamma, \omega}$.

Our strategy is as follows:

1. We show that if a wall does cross $s=m /\left(g^{\prime} n\right)$ and some other predetermined line closer to $s=y_{1} / x$ then the rank of any destabilizer must be at most $\xi x$ for a suitable $\xi$ depending only on $v$ and $X$. For $p=1$ we do not require this extra assumption and we can set $\xi=1$.

2. Assuming $F>0$, we show for any $(m, n) \in P_{\xi}$ for the $\xi$ given in (1) then any object which is $\mu_{Z}$-stable for any $Z$ in the region $\left(m /\left(g^{\prime} n\right), y_{1} / x\right) \times \mathbb{R}_{>0}$ must be a $\mu_{\omega}$-semistable sheaf.

3. We can then deduce that object must be $\mu_{Z}$-stable for all $Z$ in the region.

4. For $F=0$ we show directly that there are no walls at all.

5. We also need to assume $x>0$ but we will finish this section by showing that $y_{1}$ is always an upper bound for $R$ when $x=0$.

First of all, set $\xi=1$.

Lemma 3.3. With this notation, suppose $s \geqslant m_{0} /\left(g^{\prime} n_{0}\right)$. If $E \in \mathcal{A}_{s \omega+u \gamma, \omega}=\mathcal{A}_{s}$ is a torsion-free sheaf which is $Z_{s \omega+u \gamma, t \omega}$-stable for some $s<y_{1} / x$ and some $t>0$ then $E$ must be $\mu_{\omega}$-semistable and, for any $t>0$, each $Z_{s \omega+u \gamma, t \omega}$-destabilizing subobject of $E$ in $\mathcal{A}_{s}$ must have rank strictly bigger than $r(E)$.

Proof. Since $\frac{\tilde{y}_{1} \tilde{g}}{x} \geqslant \mu_{\omega}^{-}(E)>s g>\frac{\tilde{g} m_{0}}{n_{0}}$ and if $E$ is not $\mu_{\omega}$-semistable then $\mu_{\omega}^{-}(E)$ is given by $\mu(F)$ for some sheaf $F$ with $r(F)<r(E)$, which contradicts the definition of $m_{0}$ and $n_{0}$. So $E$ must be $\mu_{\omega}$-semistable.

Suppose that $k \rightarrow E Z_{s \omega+u \gamma, t \omega}$-destabilizes $E$ in $\mathcal{A}_{s}$ with quotient $q$ and assume that $r(K) \leqslant x$. Note that $k$ must be a sheaf $K$. We have a short exact sequence $K / Q^{-1} \rightarrow E \rightarrow Q^{0}$. Suppose $Q_{\text {free }}^{0} \neq 0$ then since $c_{1}\left(Q_{\text {free }}^{0}\right) \cdot \omega \leqslant c_{1}\left(Q^{0}\right) \cdot \omega$ and both $K / Q^{-1}$ and $Q^{0}$ are in $T$, we must have one of them with slope less than or equal to the slope of $E$. But then $K / Q^{-1} Z_{s \omega+u \gamma, t \omega}$-destabilizes $E$ for all $s<y_{1} / x$ and $t>0$ by Remark 2.2. So $Q^{0}$ must be a torsion sheaf. On the other hand, $c_{1}\left(Q^{0}\right) \cdot \omega=0$ as otherwise, $s g<\mu_{\omega}\left(K / Q^{-1}\right)<\mu_{\omega}(E)$ which is impossible by assumption. So $Q^{0}$ is supported on points. But then $Q^{-1}$ must be non-zero and so $r(K)>r(E)$.

Since $m_{0} /\left(g^{\prime} n_{0}\right)$ increases with $\xi$, this lemma also holds for any $\xi>1$ as well.

For the rank bigger than $x$ case we appeal to a different lemma. Observe first that, for any $\xi$ and $(m, n) \in P_{\xi}$ we have

$$
\frac{m}{g^{\prime} n}+\frac{2 \tilde{g} p-1}{2 g n x}=\frac{y_{1}}{x}-\frac{1}{2 g n x} .
$$

What we do now is to show that the critical wall for a destabilizing object cannot lie outside the wall given by $s=m /\left(g^{\prime} n\right)$ unless the rank is at most $r(v)$.

Lemma 3.4. Pick any $\xi>0$ and $(m, n) \in P_{\xi}$. Let $s=m /\left(g^{\prime} n\right)$. Suppose $p=1$ and $e \in \mathcal{A}_{s}$ satisfies $v(e)=v$. Then e has no subobjects $k$ in $\mathcal{A}_{s}$ such that both $\mu_{Z}(k)$ and $\mu_{Z}(e / k)$ are finite. 
Proof. Suppose $k \subset e$ in $\mathcal{A}_{s}$ such that $\mu_{Z}(k)$ and $\mu_{Z}(e / k)$ are finite. Let $\operatorname{ch}(k)=$ $\left(r, c_{1} \omega+c_{2} \gamma+\alpha^{\prime}, \chi\right)$. Since, $Z$ is a stability condition and we have $0<\operatorname{Im} Z(k)<$ $\operatorname{Im} Z(e)$ (from the assumptions on $k$ and $e / k$ ). Then

$$
0<c_{1}-r \frac{m}{g^{\prime} n}<y_{1}-x \frac{m}{g^{\prime} n}=\frac{n \tilde{y}_{1}-m x}{g^{\prime} n}=\frac{p}{g^{\prime} n} .
$$

Clearing denominators and setting $p=1$, we have $0<c_{1} g^{\prime} n-r m<1$. But $c_{1} g^{\prime} n-r m$ is an integer.

In particular, this means that, for $p=1$, no wall of finite radius can intersect $s=m /\left(g^{\prime} n\right)$. This is because, if $\mu_{Z}(e / k)$ is infinite then $k$ cannot $Z$-destabilize. If $\mu_{Z}(k)$ is infinite then $k$ must destabilize for all $t>0$ which means that $s=m /\left(g^{\prime} n\right)$ must be the wall.

Lemma 3.5. Suppose $p>1$. Let $(m, n) \in P_{\xi}$ for $\xi=\frac{2 \tilde{g} p}{2 \tilde{g} p-1}$ and let $s \geqslant$ $\frac{m_{0}}{g^{\prime} n_{0}}+\frac{2 \tilde{g} p-1}{2 g n_{0} x}$. Suppose $e \in \mathcal{A}_{s}$ satisfies $v(e)=v$. Suppose e has a subobject $k$ in $\mathcal{A}_{s}$ such that both $\mu_{Z}(k)$ and $\mu_{Z}(e / k)$ are finite. Then $0 \leqslant r(k) \leqslant r(e)$ and $0 \leqslant r(q) \leqslant r(e)$.

Proof. If the conclusion does not hold then either $r(q)$ or $r(k)$ is bigger than $x=r(e)$. Without loss of generality assume $r(k)>r(e)$ and set $c_{1}=c_{1}(k) \cdot \omega / g$ and $r=r(k)$. Since $c_{1}-r s<y_{1}-x s$, we have that $c_{1}<y_{1}+(r-x) s$ and, since $s<y_{1} / x$, this gives $c_{1}<y_{1}+(r-x) y_{1} / x=r y_{1} / x$. In other words, $\mu_{\omega}(k)<\mu_{\omega}(e)$.

But now

$$
0<c_{1}-r s \leqslant c_{1}-r\left(\frac{m_{0}}{g^{\prime} n_{0}}+\frac{2 \tilde{g} p-1}{2 g n_{0} x}\right)
$$

Rearranging gives

$$
r \frac{2 \tilde{g} p-1}{2 \tilde{g} x}<c_{1} g^{\prime} n_{0}-m_{0} r
$$

Combining with (3.1) gives

$$
r<\frac{2 \tilde{g} p}{2 \tilde{g} p-1} x=\xi x .
$$

But this is impossible for our choice of $\left(m_{0}, n_{0}\right)$.

We can now treat the $F=0$ case.

THEOREm 3.6. If $F=0$ then there are no walls in $\Pi_{\beta, \omega, y_{2} / x}$ except when $\beta \cdot \omega=$ $y_{1} g / x$.

Proof. When $F=0$, we have $C+R=y_{1} / x$ for any wall. Consequently, for any $\xi>0$, any such wall must intersect some $s=\beta \cdot \omega / g=m /\left(g^{\prime} n\right)$ for some $(m, n) \in P_{\xi}$. When $p=1$ this contradicts Lemma 3.4 (by the remark following the lemma. It must also intersect $s=\frac{y_{1}}{x}-\frac{1}{2 g n x}$ and so when $p>1$, Lemma 3.5 tells us that any object $e$ (with $v(e)=v$ ) in $\mathcal{A}_{s}$ must be a sheaf since $E^{-1}[1]$ is a subobject and $r\left(E^{-1}[1]\right)<0$ if $E^{-1}$ is non-zero. On the other hand, if a sheaf $E$ is $\mu_{Z}$-stable for all sufficiently large $t$ (if it corresponds to a wall, for example) then its torsion subsheaf must eventually 
$Z$-destabilize it (for all large enough values of $t$ ) because the $z$-slope of a torsion sheaf is $1 / t$ times a (finite) constant in $t$. So $E$ must be torsion-free. Then, by Lemma 3.3, the rank of a $Z$-destabilizing subsheaf must be bigger than $r(E)$. But that contradicts Lemma 3.5 again. In any case, for $p \geqslant 1$, there cannot be such a wall. $\square$

\section{Notes 3.7.}

1. The condition $F=0$ is a actually rather strong. It implies that $u=y_{2} / x$ (or $\gamma=0)$ and the discriminant $\Delta(E)=\alpha^{2}$. Then the Hodge Index Theorem implies that $\alpha=0$ and $\Delta(E)=0$. So these are Bogomolov critical sheaves.

2. Note that for line bundles $L$ on any smooth projective surface with $\alpha=0$ automatically satisfy $F=0$ on the plane given by $u=y_{2}$ and so we conclude they are Bridgeland stable there. This is already well known (see [AB11, Prop 3.6(b)]) in the case where $y_{2}=0$.

3. When $\rho(X)=1$ and $K_{X}=0$ then $F=0$ on a K3 surface only for line bundles but for abelian surfaces this happens exactly for semi-homogeneous bundles and we conclude that these are always $\mu_{Z}$-stable for all $Z$. This gives a proof independently of Fourier-Mukai transform methods.

4. It should be possible to repeat the analysis and these theorem for the refined basic stability condition given for K3 surfaces by Bridgeland ([Bri08] and used in [ABL07] and [MYY11a]) in which $v$ is multiplied by $\sqrt{\operatorname{Td}(X)}$. In that case the critical objects should correspond to spherical objects.

We can now assume $F>0$. Note that if $(m, n) \in P_{\xi}$ for any $\xi>0$ then we require

$$
n>\frac{p}{g^{\prime}} \sqrt{\frac{g}{\Delta}} .
$$

We now use this condition by observing that $F \geqslant \frac{\Delta}{x^{2} g}>\frac{p^{2}}{{g^{\prime}}^{2} n^{2} x^{2}}$ for all $u$ and so

$$
C<\frac{y_{1}}{x}-\sqrt{F}<\frac{y_{1}}{x}-\frac{p}{g^{\prime} n x}=\frac{m}{g^{\prime} n} .
$$

So any wall which crosses any line $s=s_{0}>m /\left(g^{\prime} n\right)$ for any $(m, n) \in P_{\xi}$ and $\xi \geqslant 1$ must also cross $s=m /\left(g^{\prime} n\right)$ as well. In particular, this holds for the least such $n$.

Now let $\xi_{p}=1$ if $p=1$ and $\xi_{p}=\frac{2 \tilde{g} p}{2 \tilde{g} p-1}$ if $p>1$.

Lemma 3.8. Let $s_{0}=m /\left(g^{\prime} n\right)$ for some $(m, n) \in P_{\xi}$ for $\xi=\xi_{p}$. Suppose e is an object of $\mathcal{A}_{s}$ for some $s_{0}<s<y_{1} / x$, where $v(e)=v$ and $r(v)>0$. Suppose for (at least) one $s$ there is a real number $t>0$ such that e is $\mu_{Z}$-stable with $Z=Z_{s \omega+u \gamma, t \omega}$. Then e must be a $\mu_{\omega}$-semistable sheaf.

Proof. The argument is the same as in the proof of Theorem 3.6.

So what we have shown is that there is some $s_{0}<y_{1} / x$ (which can be computed explicitly) with the following property. If $e$ is an object of some $\mathcal{A}_{s}$ for $s_{0}<s<y_{1} / x$ and is $\mu_{Z}$-stable for some $t$ then it must be a $\mu_{\omega}$-semistable sheaf by 3.8. But then by lemmas 3.3, 3.4 and 3.5 it must be $\mu_{Z}$-stable for all $s_{0}<s<y_{1} / x$ and $t>0$. What this means is that no wall can intersect either the region $s \geqslant \frac{m}{g^{\prime} n}$ (when $p=1$ ) or $s \geqslant \frac{2 g n y_{1}-1}{2 g n x}$ (when $p>1$ ). For the moment combine these to give the bound

$$
\frac{y_{1}}{x}-\frac{(2 p \tilde{g}-1) \epsilon+1}{2 g n x}
$$


where $\epsilon=1$ when $p=1$ and $=0$ otherwise. Then $C+R \leqslant \frac{y_{1}}{x}-\frac{(2 p \tilde{g}-1) \epsilon+1}{2 g n x}$. Solving for $C$ we obtain:

Proposition 3.9. Suppose $v$ is the Chern character of a $\mu_{\omega}$-semistable sheaf. Then the centres $C$ of the walls in $\Pi_{\beta, \omega, u}$ are bounded below by

$$
\begin{gathered}
\frac{1}{2}\left(\frac{m x+n \tilde{y}_{1}}{g^{\prime} n x}-F g^{\prime} n x\right) \text { when } p=1 \text { or } \\
\frac{4 g n y_{1}-1}{4 g n x}-\text { Fgnx when } p>1 .
\end{gathered}
$$

When $x=0$ we proceed differently. Recall from Lemma 2.4 that all walls have a fixed centre:

$$
C=\frac{z+d u y_{2}}{g y_{1}} .
$$

Observe that if $w=\left(r,\left(c_{1} \omega+c_{2} \gamma+\alpha^{\prime}\right), \chi\right)$ corresponds to a wall then $r>0$ because then $\mu_{Z}(w)-\mu_{Z}(v)$ would be constant. But using Remark 2.9, we have

$$
C<\frac{c_{1}}{r}<\frac{y_{1}}{r}+C \leqslant y_{1}+C .
$$

In other words, the slope of $w$ is bounded by constants. But, an object $e \in \mathcal{A}_{s}$ with $\operatorname{ch}(e)=w$ must satisfy $\mu_{\omega}(e)>s$ and so if the wall has radius $R=R_{v}(w)$, we have $C+R<\frac{c_{1}}{r}<y_{1}+C$ and so $R<y_{1}$. So we have proved:

Proposition 3.10. If $x=0$ then the radius of any wall for $v=\left(0, y_{1} \omega+y_{2} \gamma+\right.$ $\alpha, z)$ is less than $y_{1}$.

Combining the above results with Proposition 2.6 we have the main theorem of the paper:

TheOREm 3.11. Let $\beta$ be any class in $N(X)_{\mathbb{R}}, \omega$ an ample class and $u \in \mathbb{R}$. Fix a Chern character $v$ of a $\mu_{\omega}$-semistable sheaf or a torsion sheaf. In the half-plane $\Pi_{\beta, \omega, u}$ there are real numbers $C_{0}$ and $R_{0}$ such that all of the walls corresponding to $v$ are contained in the semi-circle with centre $\left(C_{0}, 0\right)$ and radius $R_{0}$. In particular, the radii of the walls are bounded above by $R_{0}$.

Notes 3.12. The bounds are not always sharp. This is especially true for $p>1$ where the $p=1$ bound may often suffice. But even when $p=1$, we shall see an example below where the bound is not quite sharpest. The bounds are constructive (we shall give explicit examples in the next section). For the first three points of this note we assume $p=\operatorname{gcd}\left(x, \tilde{y}_{1}\right)=1$.

1. For $(m, n) \in P_{1}$ with $n$ least

$$
\frac{1}{2}\left(\frac{m x+n \tilde{y}_{1}}{g^{\prime} n x}-F g^{\prime} n x\right) \leqslant C<\frac{y_{1}}{x}-\sqrt{F},
$$

and

$$
0<R \leqslant \frac{1}{2}\left(F g^{\prime} n x-\frac{1}{g^{\prime} n x}\right),
$$

where $F$ is given by Equation 2.1 and $g^{\prime}=g / \operatorname{gcd}_{E}\left(c_{1}(E) \cdot \omega\right)$. 
2. The upper and lower bounds on $s$ are

$$
\frac{y_{1}}{x}-F g^{\prime} n x \leqslant s<\frac{m}{g^{\prime} n} .
$$

3. Recall the construction of $m$ and $n$ : they are given by Euclid's algorithm applied to $n \tilde{y}_{1}-m x=1$ and such that there are no integers $j$ and $0<k \leqslant x$ such that

$$
\frac{m}{n}<\frac{j}{k}<\frac{\tilde{y}_{1}}{x}
$$

We also had the technical assumption on $n$ given by 3.2 but this would rarely need to be imposed as the RHS is often less than 1. It also makes sense to choose $m$ to be the least such. For example (ignoring 3.2), suppose $g^{\prime}=1$ then if $y_{1}=2$ and $x=1$, we can pick $m=1=n$. Whereas, if $y_{1}=10$ and $x=5$ then we pick $m=7$ and $n=4$.

4. The dependence on $u$ is quadratic entirely through $F$. In particular, it is potentially unbounded as $u$ increases. We shall see an example below where the walls are actually unbounded in the $(u, s)$-plane.

Combining the theorem with local finiteness of the walls we can state a version of Theorem 1.1(i) in [LQ11]:

TheOREM 3.13. Suppose $F>0$ for some $u$. Then, for an $s \neq \frac{y_{1}}{x}-\sqrt{F}$, there are only a finite number of mini-walls in the ray $\Xi_{s \omega+\gamma, \omega}$.

\section{Examples and counterexamples.}

\subsection{Rank 0 case.}

Proposition 4.1. Suppose $(X, \omega)$ is a surface with $\rho(X)=1$ and $g^{\prime}=1$. Then there are no walls in the basic Bridgeland stability plane for $v=(0,1, k g)$ for any integer $k$.

Proof. Let $E$ be a torsion sheaf with $\operatorname{ch}(E)=v$ (a twist of the structure sheaf of a divisor in the linear system of $\omega$ ) and suppose $K \rightarrow E$ is a (maximally) destabilizing object in $\mathcal{A}_{s}$ for some $s$ with $\operatorname{ch}(K)=(r, c, \chi)$. Observe that $K$ cannot have torsion because if it did $K_{\text {tors }}$ would have to have $c=1$ and so $E / K_{\text {tors }}$ would be a skyscraper and so $\mu_{Z}(K)<\mu_{K}\left(K_{\text {free }}\right)$ contradicting the maximality. So (at least) $r>0$. It also follows by a similar argument that $K$ is $\mu_{\omega}$-semistable. We use Remark 2.9. We have $C_{0}=z / g=k$ and so we have

$$
k r<c<1+k r,
$$

which is a contradiction. $\square$

When $z \neq k g$ then there may be walls. For example, consider $E=\mathcal{O}(\omega) / \mathcal{O}_{X}$ for some map $\mathcal{O}_{X} \rightarrow \mathcal{O}(\omega)$. This is just a line bundle supported on a divisor in the linear system $|\omega|$. This has Chern character $(0, \omega, g / 2)$ and there is a wall with centre $1 / 2$ and radius $1 / 2$ given by $K=\mathcal{O}(\omega)$. 
4.2. Rank 1 case. We now consider the cases studied in [MM11]. There $X=$ $(\mathbb{T}, \ell)$ was an irreducible principally polarized abelian surface (ppas for short) and so $\rho(X)=1$ and $g=2=\tilde{g}$. Note that we must have $\gamma=0$ (and so $d=0$ in he formulae of Note 3.12). We set $v=(1,2 \ell, 4-k)$ and $\beta=\omega$. Then $F=k$ and the bounds become

$$
\begin{gathered}
\frac{3-k}{2} \leqslant C<2-\sqrt{k}, \\
R \leqslant \frac{1}{2}(k-1),
\end{gathered}
$$

and the walls are constrained to satisfy $2-k \leqslant s<1$. It follows that $L^{2} \mathcal{I}_{p}$ for a point $p$ is $\sigma$-stable for all $\sigma \in \Pi_{\omega, \omega, 0}$ for which the sheaf is in $\mathcal{A}$. Note that, in that case, the maximal radius is achieved (by $w=(1, \ell, 1)$ ). This agrees with the finding of [MM11].

In that paper (and elsewhere) it is shown that the twisted ideal sheaf $L \otimes \mathcal{I}_{V}$ is $Z$-stable for any zero-scheme $V$ but the situation is very different if we assume $\mathbb{T}$ is reducible. Suppose $\mathbb{T}=E_{1} \times E_{2}$ is a product of elliptic curves (for simplicity, we assume they are non-isomorphic but this is not really necessary). Then the canonical polarization splits as $\ell=\omega=\ell_{1}+\ell_{2}$. In this case, we have $\tilde{g}=1$ and so $g^{\prime}=2$. This time we must pick $m=3$ and $n=1$. We still have $F=k$ but now $s<3 / 2$ for our walls and our maximal radius (of a pseudo-wall) is now $k-1 / 4$ with centre $7 / 4-k$. But this does not correspond to a real wall. The maximal wall is given by $w=\left(1,2 \ell_{1}+\ell_{2}, 2\right)$ with centre $2-k$ and radius $\sqrt{k(k-1)}$. Then $s<2-k+\sqrt{k(k-1)}<3 / 2$, for all $k$. In fact, the limit of $C+R$ for these walls as $k \rightarrow \infty$ is $3 / 2$ but the limit is never achieved.

To give a more complete picture, consider the same product torus but this time let $v=\left(1, \ell_{1}+\ell_{2}, 1-k\right)$. This corresponds to the sheaves $L \otimes \mathcal{I}_{V} \otimes \mathcal{P}_{\hat{x}}$, where $\mathcal{P}_{\hat{x}} \in \operatorname{Pic}^{0} \mathbb{T}$ and $|V|=k$. These sheaves have two families of potentially destabilizing subsheaves: $L_{1} \otimes \mathcal{I}_{V^{\prime}}$ and $L_{2} \otimes \mathcal{I}_{V^{\prime}}$ up to twists by flat line bundles, where $\left|V^{\prime}\right|<|V|$. These correspond geometrically to $V^{\prime} \subset V$ and $V \backslash V^{\prime}$ being contained in a translate of either $E_{2}$ or $E_{1}$, respectively. These two families of walls intersect along a plane of constant $u$ (as predicted by Theorem 3.1). The situation is illustrated in Figure 2 for the case $k=4$, where the vertical axis is $t$ and the two grey planes are $s=1$ and $u=0$. On the plane $u=0$ the walls have centres at $s=m-3$ and radii $\sqrt{m^{2}-8 m+12}$. These radii are positive for $m=0$ and $m=1$ only. Note that in the $(s, u)$ plane the walls are parabolas and so are unbounded.

4.3. Global finiteness. We can ask if the set of walls is always finite but two examples in [Mea12] show that this is not the case. For an irreducible principally polarized abelian surface $(\mathbb{T}, \omega)$ the Chern characters $(1,2 \omega, 2)$ and $(1,2 \omega, 1)$ result in infinite families of walls. But it does confirm our finding that, away from $C_{0}$, each ray contains only finitely many walls. However, we can prove:

Proposition 4.2. In the case of a principally polarized abelian surface $(\mathbb{T}, \omega)$, if $q$ is rational then the number of mini-walls in the ray $\beta=q \omega$ is finite.

Proof. This follows because for such $q$, there is a Fourier-Mukai transform $\Phi_{q}$ which reverses the ray (see [MM11, Prop 3.2]). Then the proposition follows from Theorem 3.11. $\square$ 


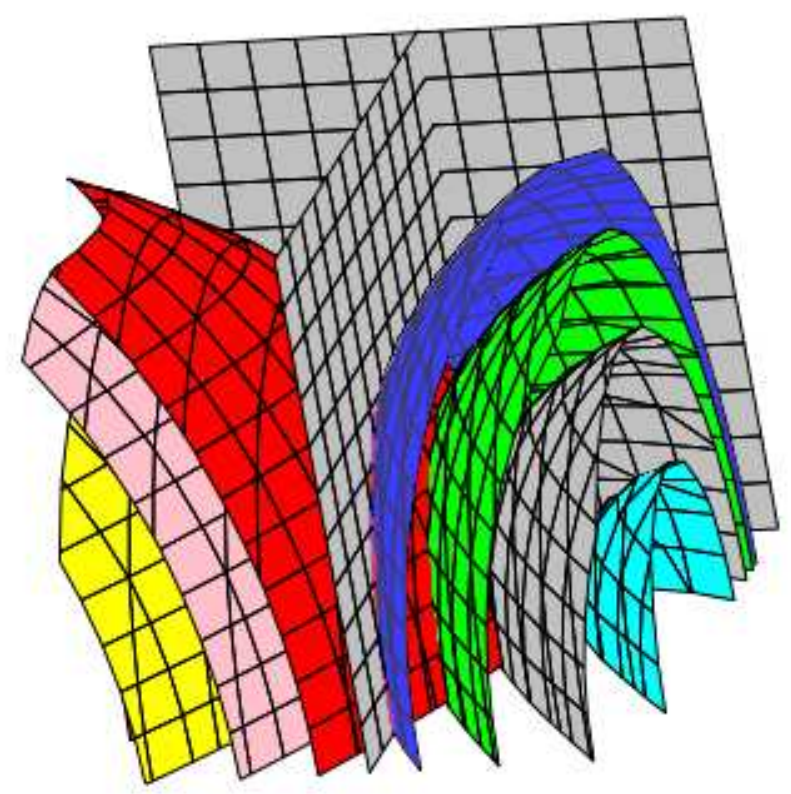

FIG. 2. Walls in $\Pi_{\ell_{1}, \ell, u}$

As was shown by Bridgeland in [Bri08], the space of all (good) Bridgeland stability conditions $\operatorname{Stab}(\mathbb{T})$ is 3-dimensional and each stability condition can be written as $\Phi(\mathcal{A}, Z)$ for some Fourier-Mukai transform $\Phi$. Since $\Phi$ preserves the chamber and wall structure we have

THEOREM 4.3. Let $(\mathbb{T}, \omega)$ be an irreducible principally polarized abelian surface. The walls in $\operatorname{Stab}(\mathbb{T})$ are nested real hypersurfaces.

REMARK 4.4. This will hold more generally for abelian surfaces and K3 surfaces when stated properly but we omit the details.

EXAMPLE 4.5. In particular, by Theorem 3.13, if $y_{1} / x-\sqrt{F}$ is rational (ie, when $F$ is a square rational) then the number of walls in $\operatorname{Stab}(X)$ is (globally) finite. For example, on a ppas $(\mathbb{T}, \omega)$ with $v=(5,3 \omega, 1)$ we have $F=4 / 25$ and then the critical ray is $s=1 / 5$.

4.4. The case of O'Grady's Holomorphic Symplectic Space. Let us now look at an example where $p \neq 1$. In [O'G03], Kieran O'Grady showed that the moduli space of Gieseker stable sheaves with Chern character $(2,0,-2)$ (modulo natural torus actions) gives a simply connected symplectic holomorphic manifold which was not one of the (then) known types.

Proposition 4.6. Let $(X, \omega)$ be an irreducible principally polarized abelian surface. Then the Chern character $v=(2,2 \omega, 0)$ has no walls in $\operatorname{Stab}(X)$. 
Proof. Since $\operatorname{Stab}(X)$ is generated by Fourier-Mukai transforms it suffices to prove this for basic Bridgeland stability conditions. Furthermore, it suffices to show that any $\mu_{\omega}$-semistable sheaf $E$ which is also $\mu_{Z}$-stable for some $Z$ is $\mu_{Z}$-stable for all $Z$. Note that $F=1$ for this choice of $v$ and $C_{0}=0$. So Remark 2.9 gives $0<c<2$ and then $c_{1}(K)=\omega$ for any destabilizing object $K$ in any $\mathcal{A}_{s}$. We also must have $r(K)>1$ because otherwise $x c_{1}-y_{1} r=0$. As usual, we can assume that $K$ is $\mu_{\omega}$-semistable. On the other hand, the centre of the wall corresponding to $(r, \omega, k)$ is $\frac{-\chi}{2 r-2}<C_{0}=0$. But then $\chi>0$ and that contradicts the Bogomolov inequality for K. $\square$

4.5. A conjecture. The examples above and Theorem 3.1 and its corollary suggest the following:

Conjecture 4.7. For any smooth complex projective surface $X$ and any $v \in$ $N(X)$ the complement of the walls in the space of good stability conditions union the boundary of its closure is path-connected.

In other words, any $\sigma$-stable moduli spaces survives to the boundary of $\operatorname{Stab}(X)$. The conjecture holds for abelian surfaces and holds for the subspace of basic stability conditions for any surface.

\section{REFERENCES}

[AB11] D. Arcara And A. Bertram, Reider's theorem and Thaddeus pairs revisited, Grassmannians, Moduli Spaces and Vector Bundles, volume 14, pp. 51-68. Clay Mathematics Proceedings, 2011.

[ABL07] D. ARCARA AND A. BERTRAM, Bridgeland-stable moduli spaces for $K$-trivial surfaces, Journal of the European Mathematical Society, 15 (2013), pp. 1-38.

[BM11] A. BAYER AND E. MACRÌ, The space of stability conditions on the local projective plane, Duke Math. J., 160 (2011), pp. 263-322.

[Bri07] T. Bridgeland, Stability conditions on triangulated categories, Ann. of Math. (2), 166 (2007), pp. 317-345.

[Bri08] T. Bridgeland, Stability conditions on K3 surfaces, Duke Math. J., 141 (2008), pp. 241-291.

[Kaw11] K. KAWATANI, Stability of Gieseker stable sheaves on K3 surfaces in the sense of Bridgeland and some applications, Kyoto Journal of Mathematics, 53 (2013), pp. 597-627.

[LQ11] J. LO AND Z. QIN, Mini-walls for Bridgeland stability conditions on the derived category of sheaves over surfaces, arxiv:1103.4352, 2011.

[Mea12] C. Meachan, Phd thesis, University of Edinburgh, 2012.

[MM11] A. MaCioCIA AND C. MeAChan, Rank one Bridgeland stable moduli spaces on a principally polarized abelian surface, International Mathematics Research Notices 2013, 9 (2013), pp. 2054-2077.

[MYY11a] H. Minamide, S. YANAGida, And K. Yoshioka, Fourier-Mukai transforms and the wall-crossing behavior for Bridgeland's stability conditions, arXiv:1106.5217, June 2011.

[MYY11b] H. Minamide, S. Yanagida, And K. Yoshioka, Some moduli spaces of Bridgeland's stability conditions, arXiv:1111.6187, November 2011.

[O'G03] K. G. O'Grady, A new six-dimensional irreducible symplectic variety, J. Algebraic Geom., 12 (2003), pp. 435-505. 
A. MACIOCIA 